12

\title{
Управление траекториями положительно и отрицательно заряженных частиц с помощью отражения на цепочке изогнутых монокристаллов
}

\author{
(C) В.А. Маишеев, М.Ю. Чесноков
}

НИЦ Курчатовский институт - ИФВЭ,

142281 Протвино, Московская обл., Россия

e-mail: Michail.Chesnokov@ihep.ru

Поступило в Редакцию 9 июля 2019 г.

В окончательной редакции 9 июля 2019 г.

Принято к публикации 25 ноября 2019 г.

Предложен быстрый алгоритм расчета прохождения частиц через цепочку изогнутых монокристаллов, основанный на явлении отражения пучка релятивистских частиц от атомных плоскостей кристалла. Результаты расчета прохождения частиц через последовательность из восьми изогнутых кристаллов сравниваются с экспериментальными данными.

Ключевые слова: изогнутый монокристалл, каналирование, объемное отражение, частицы.

DOI: 10.21883/JTF.2020.05.49190.265-19

\section{Введение}

Идеи использования каналирования частиц в изогнутых кристаллах для управления пучками были проверены и продвинуты во многих экспериментах. Однако до сих пор этот способ формирования пучка имеет ограничения в применении, так как в процессе каналирования участвуют частицы пучка с малой угловой расходимостью, ограниченной углом Линдхарда.

Недавно было открыто в экспериментах новое физическое явление - объемное отражение пучка протонов высокой энергии от изогнутых атомных плоскостей кристалла кремния $[1,2]$, которое расширяет границы применения изогнутых кристаллов на ускорителях. Это явление было предсказано в [3] в компьютерном моделировании методом Монте-Карло и позднее детально описано в аналитическом виде в работе [4].

Объемное отражение обусловлено взаимодействием налетающей релятивистской частицы с потенциалом изогнутой атомной решетки и происходит на малой длине в области, касательной к изогнутой атомной плоскости, приводя к отклонению частицы в сторону, противоположную изгибу. Вероятность отражения высока и для положительных частиц при энергиях около $100 \mathrm{GeV}$ приближается к единице. Явление отражения происходит в широкой области углов и более эффективно, чем обычное каналирование [5], поэтому имеются реальные перспективы для использования отражения при выводе и коллимации пучков на больших ускорителях.

Угол отклонения частиц при отражении от кристаллографических плоскостей для положительных частиц ограничен величиной $\theta_{v r} \approx 1.5 \theta_{c}$ (см., например, [2,4]), где $\theta_{c}=\left(2 U_{c} / p v\right)^{1 / 2}-$ критический угол каналирования, $U_{c} \sim 20 \mathrm{eV}$ - величина плоскостного канала (111) в кремнии, $p, v$ - импульс и скорость налетающей частицы. Для отрицательных частиц этот угол несколько меньше и ограничен величиной $\theta_{v r} \approx 0.8 \theta_{c}[6,4]$. Для задач ускорительной физики требуется увеличение угла отражения в несколько раз. Для этого было предложено отражение на цепочке кристаллов [7].

Принцип усиления угла отражения на цепочке кристаллов показан на рис. 1. Применяя $N \leq 10$ последовательно расположенных ориентированных кристаллов, можно увеличить угол отклонения частицы в $\sim(5-10)$ раз. Дальнейшее увеличение количества кристаллов приводит к потере эффективности процесса изза возрастания количества ядерных взаимодействий.

Предложенная идея была реализована в эксперименте [8], где получены данные по повороту пучка протонов с энергией $400 \mathrm{GeV}$ и отрицательно заряженных пионов с энергией $150 \mathrm{GeV}$.

Для оптимизации кристаллических устройств такого вида в ускорительных применениях, таких как коллимация пучка [9], или защита выводных септуммагнитов [10], требуется быстрый алгоритм расчета прохождения частиц через цепочку кристаллов. В настоящей работе такой алгоритм предложен. Результаты расчета прохождения через последовательность из восьми изогнутых кристаллов сравниваются с экспериментальными, полученными в [8].
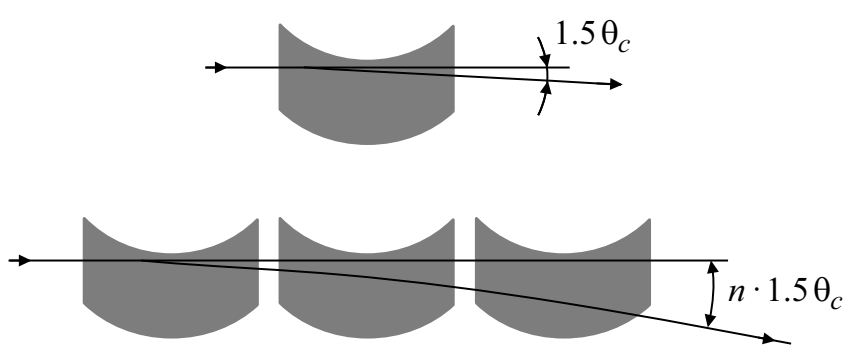

Pис. 1. Усиление угла отражения на цепочке кристаллов. 


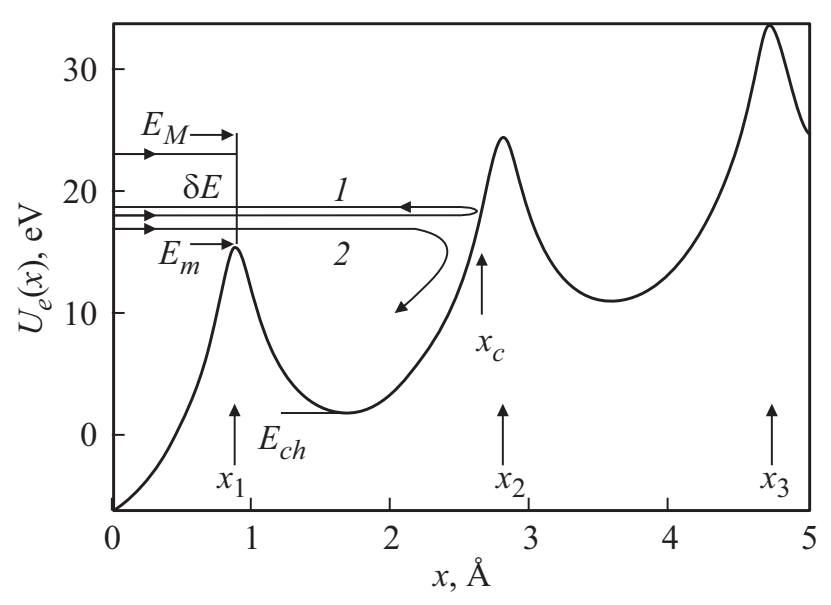

Рис. 2. Схема процессов отражения и объемного захвата: кривая показывает эффективный потенциал изогнутой кристаллографической плоскости, $x$ - поперечная координата. $E_{M}$ и $E_{m}-$ значения поперечной энергии, соответствующие соседним локальным максимумам потенциала; $E_{c h}$ - одна из поперечных энергий, соответствующих локальному минимуму потенциала; $x_{1}, x_{2}, x_{3}$ - поперечные координаты локальных максимумов.

\section{Однократное отражение на одиночном изогнутом кристалле}

Явление объемного отражения заряженных частиц в монокристаллах представляет собой когерентное рассеяние этих частиц на плоских или осевых полях изогнутых кристаллографических структур. Здесь мы рассмотрим только плоскостной случай. Наше аналитическое описание объемного отражения основано на известных уравнениях движения частиц в плоских полях изогнутых монокристаллов. В частности, для такого движения справедливо следующее уравнение [5]:

$$
\frac{E_{0} \beta^{2} v_{x}^{2}}{2 c^{2}}+U(x)+\frac{E_{0} \beta^{2} x}{R}=E,
$$

где $E_{0}, E$ и $\beta$ - полная и поперечная энергии частицы и ее скорость, деленная на скорость света $c$, $U(x)$ - периодический плоский потенциал в прямом одиночном кристалле как функция координаты, $R$ радиус изгиба кристалла, $x$ - поперечная координата. В действительности уравнение (1) описывает одномерное движение частицы в эффективном потенциале $U_{e}(x)=U(x)+E_{0} \beta^{2} x / R$. На рис. 2 показано движение частицы в таком потенциале. Видно, что частица при движении по траектории 1 в направлении возрастания эффективного потенциала подвергается отражению. Траектория 2 иллюстрирует альтернативный процесс, называемый объемным захватом в каналирование, обусловленный многократным рассеянием на атомных ядрах.

На данном этапе нашего подхода рассматривается движение заряженных частиц, не возмущенное многократным рассеянием на атомах. В соответствии с [4] угол отражения равен

$$
\begin{aligned}
\alpha(E)= & \frac{2 c}{R} \int_{x_{0}}^{x_{c}}\left[\frac{1}{\sqrt{\frac{2 c^{2}}{E_{0} \beta^{2}}\left[E-U(x)-\frac{E_{0} \beta^{2} x}{R}\right]}}\right. \\
& \left.-\frac{1}{\sqrt{\frac{2 c^{2}}{E_{0} \beta^{2}}\left[E-U\left(x_{c}\right)-\frac{E_{0} \beta^{2} x}{R}\right]}}\right] d x,
\end{aligned}
$$

где критическая точка $x_{c}$ есть решение уравнения $E-U\left(x_{c}\right)-\frac{E_{0} \beta^{2} x_{c}}{R}=0, x_{0}-$ начальная координата частицы, которая удовлетворяет уравнению:

$$
\frac{E_{0} \beta^{2} \theta^{2}}{2}+U\left(x_{0}\right)+\frac{E_{0} \beta^{2} x_{0}}{R}=E,
$$

где $\theta-$ угол входа в монокристалл. Уравнение (2) написано для симметричного случая прохождения частицы через монокристалл. В этом случае $\left|x_{0}-x_{c}\right| \approx\left|x_{e}-x_{0}\right| \approx \frac{l_{0}^{2}}{8 R}$, где $l_{0}-$ длина кристалла по пучку и $x_{e}-$ координата выхода.

Видно, что уравнение (2) описывает угол объемного отражения как функцию параметра $E$. Легко понять (вследствие периодичности $U(x)$ ), что функция $\alpha(E)$ есть периодическая функция с периодом $\delta E=E_{0} \beta^{2} d / R$. Можно найти следующее выражение для функции распределения рассеянных частиц по углам при объемномотражении:

$$
\left\langle\frac{d N}{d \alpha}(\alpha)\right\rangle=\frac{1}{\delta E} \sum_{j}\left|\frac{d \alpha}{d E}\right|^{-1}
$$

где сумма по $j$ означает, что сумма должна быть взята для каждой области однозначности функции и производная $d \alpha / d E$ должна быть рассчитана для значений $E_{j}$, которые удовлетворяют уравнению $\alpha\left(E_{j}\right)=\hat{\alpha}$, где $\hat{\alpha}$ является текущим значением угла объемного отражения.

В [4] показано, что средний угол отражения и его среднеквадратическое отклонение можно найти из уравнений

$$
\begin{gathered}
\left\langle\alpha_{v r}\right\rangle=\frac{1}{\delta E} \int_{E}^{E+\delta E} \alpha(E) d E, \\
\sigma_{v r}^{2}=\frac{1}{\delta E} \int_{E}^{E+\delta E}(\alpha(E)-\langle\alpha\rangle)^{2} d E .
\end{gathered}
$$

Здесь мы использовали результат $\left\langle\frac{d N}{d E}(E)\right\rangle=1 / \delta E$, где $N$ - число заряженных частиц (нормированное на единицу) за один период изменения поперечной энергии. Уравнения (2)-(5) были написаны для случая без возмущения многократным рассеянием. Распределение частиц на выходе из монокристалла с учетом многократного рассеяния выглядит следующим образом:

$$
\left\langle\frac{d N}{d \alpha}(\alpha)\right\rangle_{\text {exit }}=\int_{-\infty}^{+\infty} \rho_{m s}\left(\varphi, \sigma_{m s}\right)\left\langle\frac{d N}{d \alpha}(\alpha-\varphi)\right\rangle d \varphi,
$$


где $\rho_{m s}\left(\varphi, \sigma_{m s}\right)$ есть нормальное распределение, описывающее многократное рассеяние со среднеквадратичным углом $\sigma_{m s}$, соответствующее заданной длине кристалла. Легко обобщить уравнение (6) для случая пучка с произвольной угловой расходимостью вида $\rho_{b}(\alpha)$. Для среднего угла объемного отражения с конечным распределением $\rho_{R}(\alpha)$ получим

$$
\left\langle\alpha_{R}\right\rangle=\left\langle\alpha_{b}\right\rangle+\left\langle\alpha_{m s}\right\rangle+\left\langle\alpha_{v r}\right\rangle,
$$

где $\left\langle\alpha_{R}\right\rangle,\left\langle\alpha_{b}\right\rangle,\left\langle\alpha_{m s}\right\rangle$ и $\left\langle\alpha_{v r}\right\rangle$ есть средние величины распределений $\rho_{R}, \rho_{b}, \rho_{m s}$ и $\left\langle\frac{d N}{d \alpha}(\alpha)\right\rangle$ соответственно. Они рассчитываются в системе координат, где нулевой угол совпадает с входным углом $\theta$. Заметим, что $\left\langle\alpha_{m s}\right\rangle=0$ для нормального распределения. Для дисперсии конечного распределения получим соотношение

$$
\sigma_{R}^{2}=\sigma_{b}^{2}+\sigma_{m s}^{2}+\sigma_{v r}^{2},
$$

где

$$
\sigma^{2}=\int_{-\infty}^{\infty}(\alpha-\langle\alpha\rangle)^{2} \rho(\alpha) d \alpha .
$$

Характеристики $\left\langle\alpha_{v r}\right\rangle$ и $\sigma_{v r}$, полученные здесь, могут считаться невозмущенными. В нашем случае это означает, что мы не рассматриваем такой процесс, как объемный захват, в котором движущиеся частицы теряют свою поперечную энергию и захватываются в каналирование.

Для реализации описанной модели были написаны компьютерные коды [11], которые позволяют рассчитать характеристики объемного отражения положительно и отрицательно заряженных частиц в кристаллах в широком интервале энергий. Верификация кодов была проведена путем сравнения с экспериментальными данными, полученными после публикации [4]. Недавно был выполнен ряд экспериментов [12-16] по измерению среднего угла объемного отражения (рис. 3). Результаты этих измерений представлены в универсальных переменных $R / R_{0}$ по оси абсцисс и $\langle\alpha\rangle / \theta_{c}$ по оси ординат $\left(R_{0}\right.$ есть некий характерный радиус $\left.R_{0}=\beta^{2} E_{0} d / U_{0}\right)$. Видно, что экспериментальные данные хорошо согласуются с рассчитанными.

Расхождение составляет для большинства измерений менее $0.5 \mu \mathrm{rad}(<5 \%)$. Ширина углового распределения частиц при объемном захвате $\sigma_{v r}$ исследовалась в [12] для протонов $400 \mathrm{GeV}$ при разных радиусах кристалла, и также с отличием $<5 \%$ описывается представленной моделью (результаты сравнения можно найти непосредственно в [12]).

\section{Расчет прохождения пучка через последовательность кристаллов и сравнение с экспериментом}

Суть предлагаемого алгоритма состоит в том, что распределение частиц по углам после отражения в
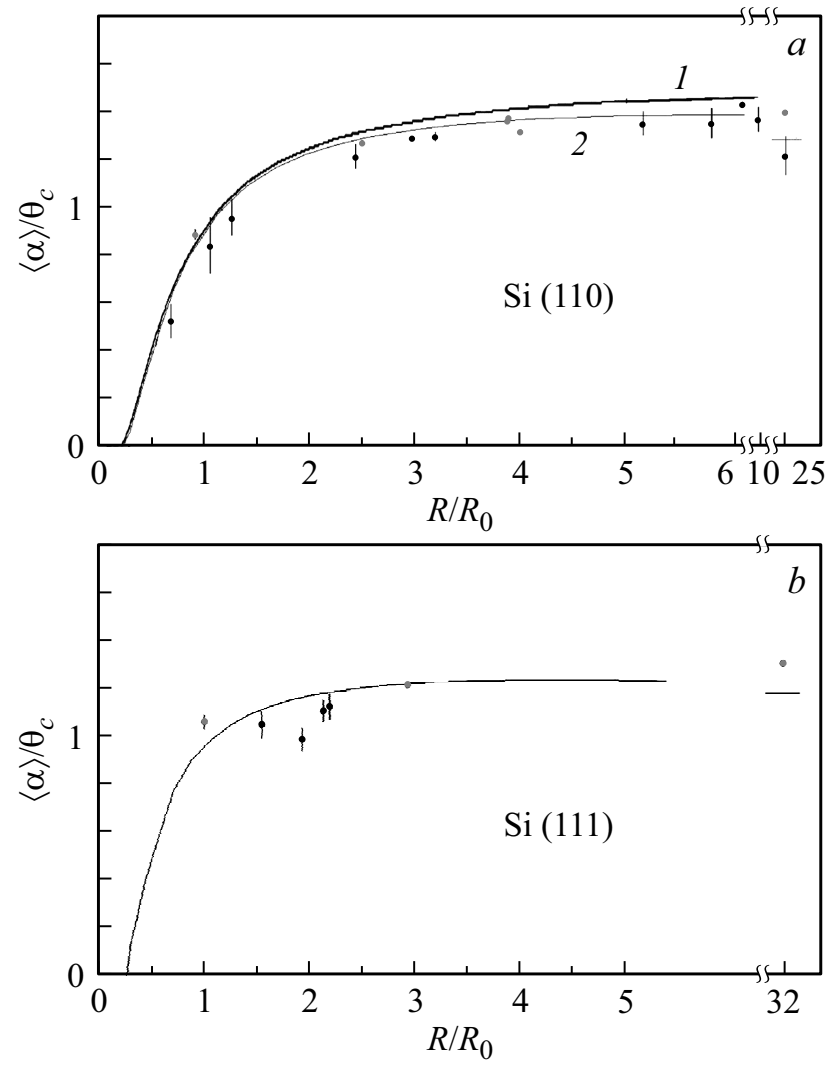

Рис. 3. $a-$ сравнение расчетных и экспериментальных величин среднего угла отражения для кристаллов (110) кремния. Кривая 1 - расчет для длинного кристалла, кривая 2 расчет для кристалла $2 \mathrm{~mm}$, использованного в опытах. Темные точки — результаты [12], серые точки — результаты [16]; $b$ - тоже для кристаллов (111) кремния. Расчет только для кристалла $2 \mathrm{~mm}$, как в экспериментах.

первом кристалле цепочки считается с применением изложенной теории по программам [11]. Распределение в каждом последующем кристалле вычисляется численно. Для этого функция распределения в одном кристалле (1 на рис. 4) разбивается на мелкие интервалы (около нескольких сотен точек), и к каждому узкому интервалу применяется процедура рассеяния в соответствии с полученным распределением для одного кристалла. Функция распределения частиц по углам в последующем кристалле получается суммированием по всем узким интервалам. Такая процедура численного интегрирования повторяется несколько раз в соответствии с количеством кристаллов в цепочке.

На рис. 4 (кривые 1) показано смоделированное распределение отклоненных протонов с энергией $400 \mathrm{GeV}$ и отрицательных пионов с энергией $150 \mathrm{GeV}$ в одном кристалле длиной $2 \mathrm{~mm}$ и радиусом изгиба $7 \mathrm{~m}$, примененном в опыте [8]. В расчетах плоскостной потенциал $U(x)$, входящий в уравнение (2), был взят из данных рентгеновских исследований для кремния [17]. Начальная угловая расходимость пучка полагалась равной нулю, что было близко к условиям эксперимента [8]. 

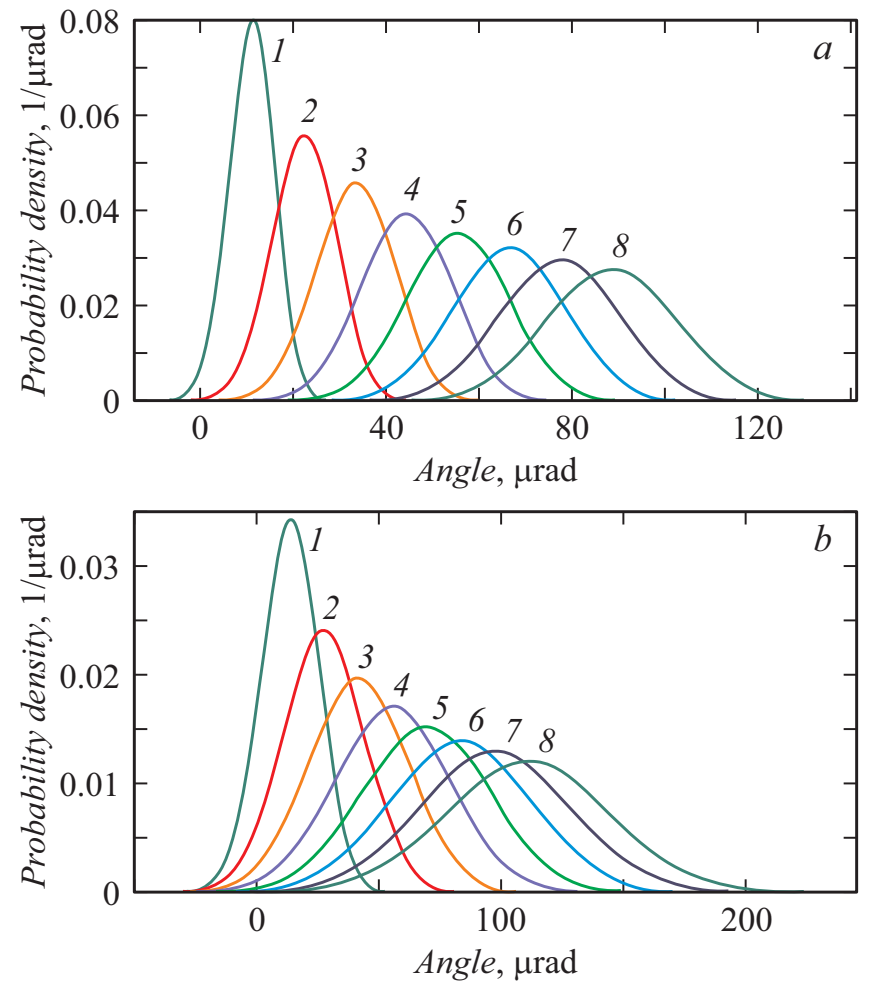

Рис. 4. $a-$ эволюция углового распределения для протонов с энергией $400 \mathrm{GeV}$ в цепочке кристаллов, где цифры над кривыми обозначают количество кристаллов; $b-$ аналогичные распределения для отрицательных пионов с энергией $150 \mathrm{GeV}$.

Расчет для отклонения в одном кристалле предсказывает для протонов средний угол отклонения $11.2 \mu \mathrm{rad}$ и среднеквадратичный размер $5 \mu \mathrm{rad}$, для отрицательных пионов - 14.3 и $11.7 \mu \mathrm{rad}$ соответственно. На рис. 4 показаны также распределения по углам частиц, отклоненных несколькими последовательно расположенными кристаллами. Видно, как происходит эволюция углового распределения частиц с увеличением количества кристаллов в цепочке.

Наконец, на рис. 5 сравниваются результаты расчета и эксперимента [8] для протонов и отрицательных пионов. Значения ширины углового распределения и угла отклонения находятся в качественном согласии. Для протонов измеренная величина $\mathrm{fwhm}=41 \mu \mathrm{rad}$, расчетная - $37 \mu \mathrm{rad}$ (отличие 10\%). Для отрицательных пионов измеренная величина fwhm $=69 \mu \mathrm{rad}$, а расчетная - $42 \mu \mathrm{rad}$ (отличие в 1.5 раза). Угол отклонения протонов в максимуме распределения в эксперименте равен $68 \mu \mathrm{rad}$, а в расчетах для последовательности из восьми кристаллов - $87 \mu \mathrm{rad}$. Для отрицательных пионов соответственно получается 78 и $112 \mu \mathrm{rad}$. Разницу угла отклонения в 20\% можно объяснить тем, что в опыте хорошо работали только 6 кристаллов из восьми. Это отчетливо видно на рис. 6, приведенном в работе [8].

На этом рисунке область углов, соответствующих отражению, показана черной стрелкой $\left(\Phi_{x} \approx 200 \mu \mathrm{rad}\right)$, при этом частицы отклоняются вниз. Сверху видны пики, соответствующие каналированию. Видно, что два пика, помеченные символом (*), „выбиваются“ из остальных по угловому положению. Это значит, что эти два кристалла разориентированы относительно остальных и
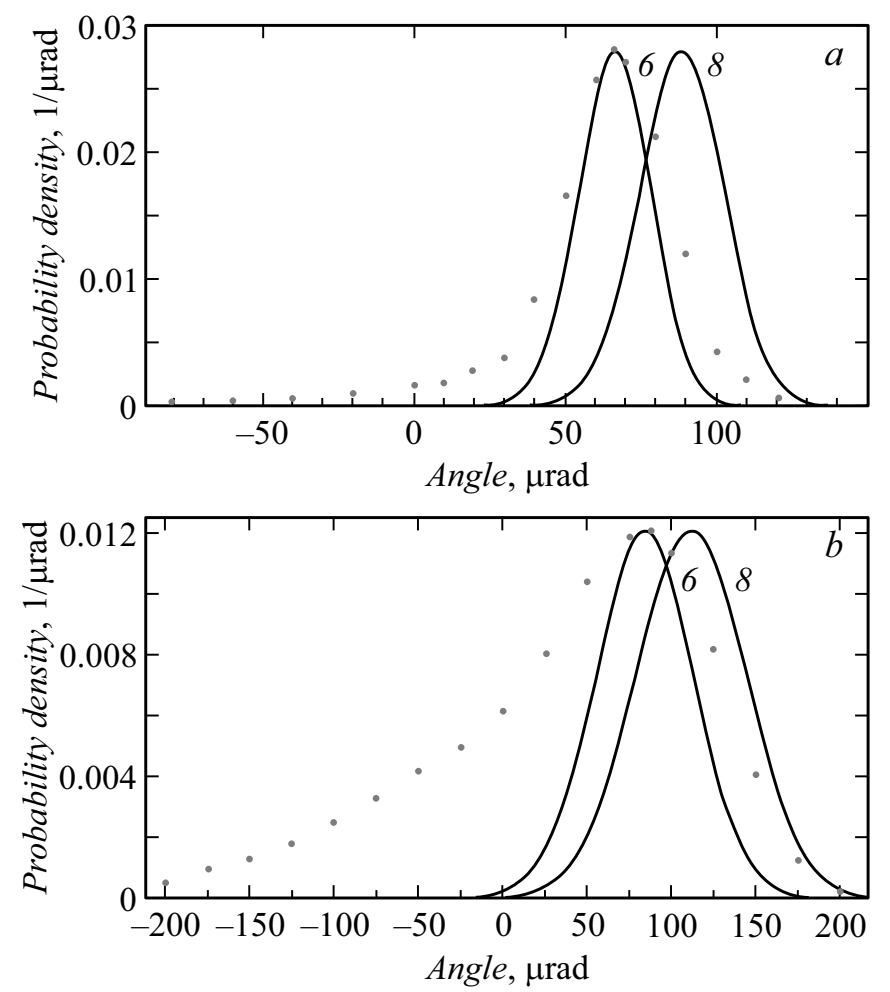

Рис. 5. $a-$ угловые распределения на выходе из цепочки для протонов, точки - эксперимент, кривая $8-$ расчет для 8 кристаллов, кривая $6-$ расчет для 6 кристаллов; $b-$ аналогичные данные для отрицательных пионов.

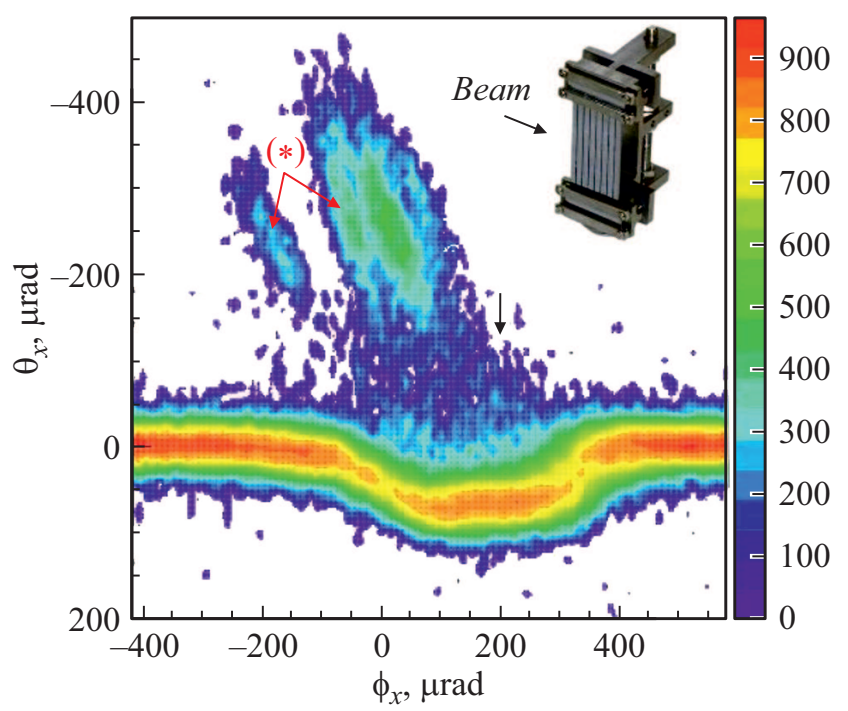

Рис. 6. Зависимость углов отклонения протонов $\theta_{x}$ от угла вращения $\Phi_{x}$ в гониометре кристаллического устройства из восьми изогнутых полосок, показанного вверху справа на вставке. 
не дают полноценного вклада в процесс отражения при ориентации кристаллического устройства, помеченного черной стрелкой, поэтому на рис. 5 мы привели также результаты расчета для последовательности из шести кристаллов, и тогда углы отклонения частиц в максимуме распределения совпали с экспериментом с точностью до $1 \%$.

Также предложенная модель не объясняет уширения и хвоста распределения в отрицательную сторону для отрицательно заряженных пионов. Рассеяние в отрицательную сторону углов может объясняться процессом „объемного захвата“ в каналирование, который мал для положительных частиц, всего $2 \%$ для $400 \mathrm{GeV}$ протонов (по данным работы [2]), и составляет $20 \%$ для $150 \mathrm{GeV}$ пионов по измерениям [6]. Для отрицательных частиц планируется разработать более точную модель прохождения частиц в режиме отражения, учитывающую альтернативный процесс объемного захвата в каналирование.

\section{Заключение}

На данном этапе предложенный алгоритм для прохождения частиц через многослойные кристаллические структуры хорошо описывает поведение протонов и будет применяться в задачах оптимизации кристаллических устройств для защиты септума СМ24 и коллимации пучка протонов кристаллами в магнитном блоке 86 ускорителя У70 [10]. Также многослойные кристаллические структуры могут быть применены для защиты электростатического септума ускорителя СПС в ЦЕРН [18], для чего с помощью предложенного алгоритма будут проведены соответствующие расчеты.

Что касается применения изогнутых кристаллов для управления пучками отрицательных частиц, пока, насколько известно, ускорительных задач не рассматривается. Но если такие появятся, то отклонение пучков отрицательно заряженных частиц последовательностью изогнутых кристаллов представляется перспективным способом, поскольку здесь достигнуты эффективности отклонения 70\% [8] при углах отклонения $100 \mu \mathrm{rad}$, a альтернативный способ отклонять электроны с помощью каналирования в коротких кристаллах показал эффективность только 30\% [19].

\section{Финансирование работы}

Работа поддержана Российским научным фондом (грант 17-12-01532).

\section{Конфликт интересов}

Авторы заявляют, что у них нет конфликта интересов.

\section{Список литературы}

[1] Ivanov Yu.M., Petrunin A.A., Skorobogatov V.V., Gavrikov Yu.A., Gelamkov A.V., LP Lapina L.P., Schetkovsky A.I., Vavilov S.A., Baranov V.I.,Chesnokov Yu.A., Afonin A.G., Baranov V.T., Chepegin V.N., Guidi V., Scandale W., Vomiero A. // Phys. Rev. Lett. 2006. Vol. 97. P. 144801.

[2] Scandale W., Still D., Carnera A., Della Mea G., De Salvador D., Milan R., Vomiero A., Baricordi S., Dalpiaz P., Fiorini M., Guidi V., Martinelli G., Mazzolari A., Milan E., Ambrosi G., Azzarello P., Battiston R., Bertucci B., Burger W.J., Ionica M., Zuccon P., Cavoto G., Santacesaria R., Valente P., Vallazza E., Afonin A.G., Baranov V.T., Chesnokov Yu.A., Kotov V.I., Maisheev V.A., Yaznin I.A., Afansiev S.V., Kovalenko A.D., Taratin A.M., Denisov A.S., Gavrikov Yu.A., Ivanov Yu.M., Ivochkin V.G., Kosyanenko S.V., Petrunin A.A., Skorobogatov V.V., Suvorov V.M., Bolognini D., Foggetta L., Hasan S., Prest M. // Phys. Rev. Lett. 2007. Vol. 98. P. 154801.

[3] Taratin A.M., Vorobiev S.A. // Phys. Lett. A. 1987. Vol. 119. P. 425.

[4] Maisheev V.A. // Phys. Rev. ST Accel. Beams. 2007. Vol. 10. P. 084701.

[5] Biryukov V.M., Chesnokov Yu.A., Kotov V.I. Crystal Channeling and Its Application at High-Energy Accelerators. Berlin: Springer, 1997.

[6] Scandale W., Vomiero A., Bagli E., Baricordi S., Dalpiaz P., Fiorini M., Guidi V., Mazzolari A., Vincenzi D., Milan R., Della Mea G., Vallazza E., Afonin A.G., Chesnokov Yu.A., Maisheev V.A., Yazynin I.A., Golovatyuk V.M., Kovalenko A.D., Taratin A.M., Denisov A.S., Gavrikov Yu.A., Ivanov Yu.M., Lapina L.P., Malyarenko L.G., Skorobogatov V.V., Suvorov V.M., Vavilov S.A., Bolognini D., Hasan S., Mattera A., Prest M., Shiraishi S. // Phys. Lett. B. 2009. Vol. 681. P. 233.

[7] Scandale $W$., Vomiero A., Baricordi S., Dalpiaz P., Fiorini M., Guidi V., Mazzolari A., Della Mea G., Milan R., Ambrosi G., Zuccon P., Bertucci B., Burger W., Duranti M., Cavoto G., Santacesaria R., Valente P., Luci C., Iacoangeli F., Vallazza E., Afonin A.G., Chesnokov Yu.A., Kotov V.I., Maisheev V.A., Yazynin I.A., Kovalenko A.D., Taratin A.M., Denisov A.S., Gavrikov Yu.A., Ivanov Yu.M., Lapina L.P., Malyarenko L.G., Skorogobogatov V.V., Suvorov V.M., Vavilov S.A., Bolognini D., Hasan S., Mozzanica A., Prest M. // Phys. Rev. Lett. 2009. Vol. 102. P. 084801.

[8] Scandale W., Arduini G., Butcher M., Cerutti F., Garattini M., Gilardoni S., Lechner A., Losito R., Masi A., Mereghetti A., Metral E., Mirarchi D., Montesano S., Redaelli S., Rossi R., Schoofs P., Smirnov G., Bagli E., Bandiera L., Baricordi S., Dalpiaz P., Germogli G., Guidi V., Mazzolari A., Vincenzi D., Claps G., Dabagov S., Hampai D., Murtas F., Cavoto G., Iacoangeli F., Ludovici L., Santacesaria R., Valente P., Galluccio F., Afonin A.G., Chesnokov Yu.A., Durum A.A., Maisheev V.A., Sandomirskiy Yu.E., Yanovich A.A., Kovalenko A.D., Taratin A.M., Gavrikov Yu.A., Ivanov Yu.M., Lapina L.P., Ferguson W., Fulcher J., Hall G., Pesaresi M., Raymond M., Bolognini D., Hasan S., Prest M., Vallazza E. // JETP. Lett. 2015. Vol. 101. P. 679.

[9] Afonin A.G., Baranov V.T., Gorlov V.N., Kotov V.I., Maisheev V.A., Terekhov V.I., Chepegin V.N., Chesnokov Yu.A., Yazynin I.A. // Atom. Ener. 2009. Vol. 106. P. 409. 
[10] Afonin A.G., Barnov E.V., Britvich G.I., Chesnokov Yu.A., Chirkov P.N., Durum A.A., Kostin M.Yu., Lobanov I.S., Maisheev V.A., Poluektov I.V., Reshetnikov S.F., Sandomirskiy Yu.E., Savin D.A., Yanovich A.A. // Phys. Rev. AB. 2019. Vol. 22. P. 033001.

[11] Program for calculation of volume reflection in bent crystals: http://mail.ihep.ru/ maisheev

[12] Scandale W., Vomiero A., Baricordi S., Dalpiaz P., Fiorini M., Guidi V., Mazzolari A., Milan R., Della Mea G., Ambrosi G., Bertucci B., Burger W., Duranti M., Zuccon P., Cavoto G., Iacoangeli F., Luci C., Pisano S., Santacesaria R., Valente P., Vallazza E., Afonin A.G., Chesnokov Yu.A., Kotov V.I., Maisheev V.A., Yazynin I.A., Kovalenko A.D., Taratin A.M., Denisov A.S., Gavrikov Yu.A., Ivanov Yu.M., Lapina L.P., Malyarenko L.G., Skorobogatov V.V., Suvorov V.M., Vavilov S.A., Bolognini D., Hasan S., Lietti D., Mozzanica A., Prest M. // Phys. Rev. Lett. 2008. Vol. 101. P. 234801.

[13] Scandale W., Carnera A., Della Mea G., De Salvador D., Milan R., Vomiero A., Baricordi S., Dalpiaz P., Fiorini M., Guidi V., Martinelli G., Mazzolari A., Milan E., Ambrosi G., Azzarello P., Battiston R., Bertucci B., Burger W., Ionica M., Zuccon P., Cavoto G., Santacesaria R., Valente P., Vallazza E., Afonin A.G., Baranov V.T., Chesnokov Yu.A., Kotov V.I., Maisheev V.A., Yazynin I.A., Afanasiev S.V., Kovalenko A.D., Taratin A.M., Denisov A.S., Gavrikov Yu.A., Ivanov Yu.M., Ivochkin V.G., Kosyanenko S.V., Petrunin A.A., Skorobogatov V.V., Suvorov V.M., Bolognini D., Foggetta L., Hasan S., Prest M. // Phys. Rev. STAccel. Beams. 2008. Vol. 11. P. 063501.

[14] Scandale $W$., Vomiero A., Bagli E., Baricordi S., Dalpiaz P., Fiorini M., Guidi V., Mazzolari A., Vincenzi D., Milan R., Della Mea G., Vallazza E., Afonin A.G., Chesnokov Yu.A., Maisheev V.A., Yazynin I.A., Golovatyuk V.M., Kovalenko A.D., Taratin A.M., Denisov A.S., Gavrikov Yu.A., Ivanov Yu.M., Lapina L.P., Malyarenko L.G., Skorobogatov V.V., Suvorov V.M., Vavilov S.A., Bolognini D., Hasan S., Mattera A., Prest M., Tikhomirov V.V. // Phys. Lett. B. 2009. Vol. 682. P. 274.

[15] Hasan S. , Bolognini D., Dalpiaz P., Della Mea G., De Salvetor D., Fiorini M., Guidi V., Mazzolari M., Milan R.,Lietti D., Prest M., Vallazza E. // Nucl. Instrum. Methods Phys. Res. Sect. B. 2011. Vol. 269. P. 612.

[16] Rossi R., Cavoto G., Mirarchi D., Redaelli S., Scandale W. // Nucl. Instrum. Meth. B. 2015. Vol. 355. P. 369.

[17] Bagli E., Guidi V., Maisheev V.A. // Phys. Rev. E. 2010. Vol. 81. P. 026708.

[18] Velotti F.M., Bestmann P., Butcher M., Calviani M., Di Castro M., Donze M., Esposito L.S., Fraser M.A., Gilardoni S.S., Goddard B., Kain V., Lendaro J., Masi A., Mirarchi D., Pari M., Prieto J.P., Redaelli S., Rossi R., Scandale W., Galvez P.S., Seidenbinder R., Stoel L.S., Zamantzas C., Zhovkovska V., Addesa F., Iacoangeli F., Galluccio F., Murtas F., Afonin A.G., Chesnokov Yu.A., Durum A.A., Maisheev V.A., Sandomirskiy Yu.E., Yanovich A.A., Garattini M., Borg J., Hall G., James T., Pesaresi M., Denisov A.S., Gavrikov Yu.A., Ivanov Yu.M., Koznov M.A., Malyarenko L.G., Skorobogatov V.V. // IPAC-2019 http://accelconf.web.cern.ch/AccelConf/ipac2019/papers/thxxplm2.pdf

[19] Wienands U., Markiewicz T.W., Nelson J., Noble R.J., Turner J.L., Uggerhoj U.I., Wistisen T.N., Bagli E., Bandiera L., Germogli G., Guidi V., Mazzolari A., Holtzapple R., Miller M. // Phys. Rev. Lett. 2015. Vol. 114. P. 074801. 